\title{
Envelhecimento e dança: análise da produção científica na Biblioteca Virtual de Saúde
}

\author{
Aging and dance: analysis of scientific production in the Virtual Health Library
}

\section{Resumo}

O envelhecimento produz perda progressiva das aptidões funcionais e da integração social, e a dança vem contribuir na melhora e/ou manutenção dessas perdas, refletindo positivamente na qualidade de vida dos idosos. Pelo fato de a produção científica ser um processo contínuo que traz a dinâmica da descoberta, contribuindo com a ciência e fortalecendo o ensino e o saber científico, desenvolveu-se esta pesquisa, com o objetivo de realizar uma análise da produção científica sobre a temática dança e o idoso nas bases de dados LILACS, MEDLINE e SciELO acessadas pela Biblioteca Virtual de Saúde (BVS), especificando: tipo de publicação, título, autoria e gênero, estrutura discursiva dos resumos, tipo de pesquisa, estratégias e tipo de análise. Os descritores utilizados foram dança e idoso(s) e dança e envelhecimento. Foram levantados 82 artigos; mas 14 artigos foram excluídos devido a ausência de resumo, totalizando 68 artigos para a análise. Os resultados metacientíficos revelaram que: 92,64\% eram artigos; melhor adequação do título quanto ao número de vocábulos; 89,9\% publicações de autoria múltipla produzida por ambos os gêneros; estrutura dos resumos adequada; as pesquisas de campo (82,35\%), descritivas $(57,36 \%)$ e a análise quantitativa $(45,6 \%)$ são as mais utilizadas. Conclui-se que os resumos estão adequados aos parâmetros metacientíficos, exceto pelo título, porém há necessidade de novas pesquisas sobre o tema com delineamentos experimentais baseados em evidências e análises inferências dos resultados para o avanço do conhecimento sobre dança e envelhecimento.

\section{Abstract}

Aging produces progressive loss of the functional aptitudes and social integration, and dance helps improve and / or maintain such losses, contemplating positively in the elderly quality of life. Scientific production is a continuous process that brings the dynamics of discovery, contributing with science and strengthening teaching and scientific knowledge, so this research was developed with the aim of accomplishing an analysis of the scientific production on the theme of dance and elderly in the
Palavras-chave: Idoso. Música. Dança. Qualidade de Vida. Revisão. Metaciência.

Programa de Pós-graduação em Ciências do Envelhecimento. Universidade São Judas Tadeu. São Paulo, SP, Brasil.

\section{Carla Witter ${ }^{1}$ \\ Marcelo de Almeida Buriti ${ }^{1}$ Gleice Branco Silva' Renatta Simões Nogueira ${ }^{1}$ Eliane Florêncio Gama ${ }^{1}$}


Virtual Health Library's database, namely: kind of publication, title, authorship and genre, discursive structures of the summaries, research type, strategies and analysis type. The descriptors used were "dance and elderly" and "dance and aging". We surveyed 82 articles; however, 14 articles were excluded due to summary absence, totaling 68 articles for the analysis. Meta-scientific results show that: $92.64 \%$ were articles; more appropriate title for the number of words, $89.9 \%$ produced multiple publications authored by both genders, adequate structure of abstracts, field research $(82.35 \%)$, descriptive (57.36\%) and quantitative analysis (45.6\%) are most often used. The conclusion is that the abstracts are adequate to meta-scientific parameters, except for the title, but there is need for further research on the theme with experimental designs based on evidence and inferences analysis results for the advancement of knowledge on dance and aging.

\section{INTRODUÇÃO}

A produção científica é um processo contínuo que traz a dinâmica da descoberta, contribuindo com o país, com a ciência e, sobretudo, fortalecendo o ensino e o saber científico. Ciência requer observação da realidade, criticidade, análise, aperfeiçoamento, novos métodos, investigação, ou seja, as pesquisas são importantes para a ordenação do mundo, para ampliação do conhecimento, tendo como principal objetivo o favorecimento dos diversos setores econômico, político, social e psicológico. ${ }^{1}$

As projeções demográficas revelam o considerável aumento da população idosa em todo o mundo, com estimativa de que, no ano de 2025 , aproximadamente $15 \%$ da população brasileira será composta por pessoas acima de 60 anos. Paralelamente, observa-se uma transição epidemiológica, expressa pelo crescente aumento de doenças crônicas degenerativas nesta faixa etária, aumentando também a necessidade de serviços de atenção à saúde dos idosos. ${ }^{2}$ As doenças diagnosticadas em um indivíduo idoso, se não forem devidamente tratadas e acompanhadas ao longo dos anos, tendem a apresentar complicações e sequelas que comprometem a independência e a autonomia do paciente. A saúde não é mais medida pela presença ou não de doenças, e sim pelo grau de preservação da capacidade funcional. ${ }^{3}$

O envelhecimento produz uma perda progressiva das aptidões funcionais do organismo, e essas alterações acabam por limitar
Key words: Elderly. Music. Dancing. Quality of Life. Review. Metascience. as capacidades do idoso em realizar suas atividades habituais. A imagem que se configura a partir disso é de incapacidade, adotando a postura de dependência, dando origem a sentimentos de inutilidade e solidão que conduz à perda gradativa das relações afetivas e sociais. Dessa forma, o envelhecimento populacional se traduz em maior carga de doenças e incapacidades, refletindo na maior procura dos idosos por serviços de saúde. ${ }^{4}$ É necessário, portanto, melhorar a qualificação dos profissionais pertencentes à equipe multiprofissional, que trabalham direta ou indiretamente com este público, a fim de tentar minimizar os impactos inerentes a este período da vida, proporcionando um envelhecimento bem-sucedido com qualidade de vida. ${ }^{5}$

O bem-estar na velhice, ou saúde num sentido amplo, seria o resultado do equilíbrio entre as várias dimensões da capacidade funcional do idoso, sem necessariamente significar ausência de problemas em todas as dimensões. ${ }^{6}$ Neste sentido, as vantagens da prática de exercícios físicos para idosos dependem de como se processa o envelhecimento e da rotina praticada. Um bom programa de exercícios para idosos deve enfatizar a flexibilidade, resistência aeróbica e força para a manutenção da massa muscular.7 Intervenções devem ser adaptadas aos desejos do indivíduo, suas preferências e necessidades, de modo a auxiliar na manutenção da atividade física em idosos adultos, sedentários e ativos. ${ }^{8}$ Neste contexto, a dança vem contribuir significativamente na melhora e/ou manutenção de várias dimensões que estão inseridas no termo 
"qualidade de vida", entre elas a integração social, a adaptação às condições preexistentes e a aptidão funcional. ${ }^{9}$

A dança é uma forma expressiva de movimentos guiados pela música. Dançar desperta emoções positivas, prazer e socialização. São esses fatores que motivam o indivíduo a dançar e os mantêm empenhados na atividade. ${ }^{10}$ Somente nos últimos 100 anos, apareceram os estudos sistemáticos sobre a imagem corporal e a dança, destacando sua atuação no processo biopsicossocial, dentro do âmbito da promoção à saúde, de um envelhecimento ativo e saudável. ${ }^{11}$

O objetivo geral foi realizar uma revisão sistemática das produções científicas publicadas nas bases de dados LILACS, MEDLINE e SciELO, acessadas pela Biblioteca Virtual de Saúde, sobre a temática da dança e idoso. Especificamente, objetivou-se avaliar as seguintes variáveis: tipo de publicação; número de vocábulos; autoria (única, coautoria, múltipla) e gênero (masculino, feminino, indefinido); estrutura discursiva dos resumos; tipo de pesquisa; estratégias e tipo de análise de dados.

\section{METODOLOGIA}

O material utilizado na pesquisa foi constituído pelos resumos de 68 artigos, resultantes de consultas nas bases de dados LILACS, MEDLINE e SciELO, acessadas pela Biblioteca Virtual de Saúde, datando o mais antigo de $1983^{12}$ e os mais recentes de 2010. ${ }^{13}$ A Biblioteca Virtual de Saúde foi escolhida tendo em vista a diversidade de periódicos indexados, permitindo assim acesso a uma gama de estudos sobre o tema proposto. Não foram utilizados limitadores temporais. Dessa forma, todo o conteúdo das bases consultadas contendo as palavras utilizadas para a busca foi contemplado. Foram levantados resumos nacionais e internacionais. Foi utilizada uma ficha de registro atendendo aos objetivos específicos propostos.

Para a coleta de dados, foram utilizadas as palavras-chave "dança e idoso(s)" e "dança e envelhecimento". Inicialmente, foram capturados
82 artigos na base de dados, eliminando os artigos repetidos e discrepantes que não atendiam ao objetivo do estudo. Deste total, entretanto, foram excluídos 14 estudos devido à ausência de resumo, perfazendo 68 artigos para a análise.

\section{RESULTADOS E DISCUSSÃO}

Os resultados quanto ao tipo de publicação foram definidos em três categorias: artigos científicos, monografias e teses. Dos 68 trabalhos analisados, 63 apresentaram-se em artigos de diversos periódicos correspondendo a 92,64\% da amostra. Como exemplo de texto publicado em periódico, encontra-se Effect of the exercise dance for seniors program on lower-body functioning among institutionalized older adults. ${ }^{13} \mathrm{Na}$ categoria monografia, apresentaram-se dois trabalhos $(2,94 \%)$ e três teses, representando $4,41 \%$. A alta concentração em periódicos permite afirmar que há uma publicação sistemática sobre o tema, revelando seu desenvolvimento científico. ${ }^{14}$

Esta consideração decorre do fato de os periódicos serem a principal e mais atualizada fonte de informação científica. Por essa razão, espera-se que seja o suporte principal de comunicação dos trabalhos científicos. Além disso, tendem a dar maior visibilidade do produto, e a avaliação da produção de áreas desenvolvidas mostra que o fato de se contar com um número significativamente maior de textos publicados em revistas científicas é um bom indicador de desenvolvimento da área. ${ }^{14,15}$

As pesquisas de produção científica sobre estresse em estudantes universitários e depressão em adolescentes apresentaram resultados semelhantes, com a maior parte da divulgação dos trabalhos concentrada em artigos (68,6\% e $98,24 \%$ ), demonstrando que os periódicos são os suportes mais utilizados para a divulgação de pesquisas, sendo um meio de disseminação do conhecimento renovado com frequência e com controle de qualidade feita pelos pares. ${ }^{14-16}$

O título é o primeiro contato do leitor com o texto e deve seguir normas científicas e éticas que recomendam que o autor elabore o título de 
seu trabalho (artigo, dissertação, tese) com até, no máximo, 12 vocábulos. Os dados mostram que $54,4 \%$ dos trabalhos analisados respeitaram a recomendação e 45,6\% não levaram em consideração as recomendações propostas na construção dos títulos das pesquisas. Um exemplo adequado é o trabalho intitulado Dancer's perceptions of paint and injury: positive and negative effects. ${ }^{17}$ Outro artigo, com o título Effect of a community based Argentine tango dance program on functional balance and confidence in older adults, ${ }^{18}$ encontra-se inserido nas pesquisas que não levaram em consideração as recomendações para construção dos títulos, assim como o artigo Waltr dancing in patients with chronic heart failure: new form of exercise training, com 13 vocábulos. ${ }^{19}$ Foi aplicado o qui-quadrado $\left(\chi_{o}^{2}=0,53\right.$ e $\chi_{c}^{2}=$ $3,84$, n.g.l. $=1$ e $\mathrm{p} \leq 0,05)$, que revelou não existir diferença estatisticamente significante, o que é indício da necessidade de orientadores e orientandos estarem mais atentos aos títulos que atribuem as publicações. Os resultados indicam a necessidade de um desenvolvimento mais bem sustentado na área.
A pesquisa sobre Psicologia Forense/Jurídica no banco de dissertações e teses da Capes encontrou resultados semelhantes, estando $44,11 \%$ dos títulos de dissertações de mestrado e teses de doutorado de acordo com as normas científicas de elaboração. ${ }^{1} \mathrm{~A}$ análise do descritor leitura na revista Reading Research Quarterly revelou que a média de vocábulos por título foi 10,02, estando dentro do padrão das normas científicas, atribuindo este resultado à qualificação da revista, sendo uma das mais conceituadas na área de leitura com alto fator de impacto. ${ }^{20}$

A tabela 1 apresenta os resultados quanto ao número de autores responsáveis pelo trabalho, sendo dividida em três categorias: autoria única, coautoria e autoria múltipla (89,9\%), e o gênero foi dividido em masculino, feminino e indefinido. Como exemplo, cita-se o trabalho de Miriam Aparecida Herrera Fernandes, intitulado Intervenção em instituição de idosos: ouvir músicas, dançar, escutar histórias, brincar, criar personagens, em que foi realizado um lançamento na categoria única e no gênero feminino. ${ }^{21}$

Tabela 1 - Autoria e gênero nos artigos sobre dança e envelhecimento acessados pela Biblioteca Virtual de Saúde. São Paulo, SP, 2011.

\begin{tabular}{ccccccccc}
\hline & \multicolumn{1}{c}{ Gênero } \\
\cline { 2 - 10 } Autoria & \multicolumn{2}{c}{ Masculino } & \multicolumn{2}{c}{ Feminino } & \multicolumn{2}{c}{ Indefinido } & \multicolumn{2}{c}{ Total } \\
& $\mathrm{N}$ & $\%$ & $\mathrm{~N}$ & $\%$ & $\mathrm{~N}$ & $\%$ & $\mathrm{~N}$ & $\%$ \\
Única & 1 & 5 & 6 & 13 & 7 & 3,66 & 14 & 5,45 \\
Coautoria & 0 & 0 & 0 & 0 & 12 & 6,28 & 12 & 4,67 \\
Múltipla & 19 & 95 & 40 & 87 & 172 & 90,1 & 231 & 89,9 \\
\hline Total & 20 & 100 & 46 & 100 & 191 & 100 & 257 & 100 \\
\hline
\end{tabular}

Para o trabalho de Murrock \& Madigan, Sef-efficacy and social support as mediators between culturally specific dance and lifestyle physical activity, realizou-se um registro na categoria coautoria e no gênero indefinido, pois os pré-nomes estavam abreviados. $^{22}$ Para Rohleder, Beulen, Chen, Wolf \& Kirschbaum, intitulado Stress on the floor: the cortisol stress response to social evaluative threat in ballroom dancers, realizou-se o registro na categoria múltipla e no gênero indefinido. ${ }^{23}$

O teste estatístico $\left(\chi_{\mathrm{o}}^{2}=369,85\right.$ e o $\chi_{\mathrm{c}}^{2}=5,99$, n.g.l. $=2$ e $\mathrm{p} \leq 0,05)$ demonstrou a existência de significância estatística, com predomínio de autorias múltiplas. Esse resultado demonstra que as pesquisas analisadas estão seguindo a tendência 
científica atual. Quanto ao gênero, observa-se a presença de homens e mulheres na realização de pesquisas e, consequentemente, de ciência, porém muitos pré-nomes estavam abreviados, o que não permitiu uma análise mais consistente. A pesquisa de produção científica sobre Depressão em Adolescentes obteve resultados similares, com predomínio de autorias múltiplas $(87,5 \%)$ e sem especificação de gênero. ${ }^{14}$

Outro aspecto estudado foi o resumo, parte importante do texto que é inserida na base de dados e que deve conter informações que ofereçam ao leitor uma síntese global do trabalho.
Deve ser suficientemente claro, explícito e ter todas as informações essenciais para o leitor poder tomar decisão de continuar sua busca e leitura integral do material. A tabela 2 apresenta os resultados, mas algumas observações de caráter qualitativo merecem ser feitas: alguns resumos estavam muito aquém em termos de extensão e apenas enunciavam um ou outro aspecto do trabalho; outros foram redigidos com falhas de digitação e consumindo a maior parte do espaço com a introdução, sobrando quase nada para partes essenciais do trabalho, como o método e os resultados. ${ }^{20}$

Tabela 2 - Estrutura discursiva dos resumos dos artigos sobre dança e envelhecimento acessados pela Biblioteca Virtual de Saúde. São Paulo, SP, 2011.

\begin{tabular}{ccccccccccccc}
\hline & \multicolumn{2}{c}{ Frase inicial } & \multicolumn{2}{c}{ Objetivo } & \multicolumn{2}{c}{ Participantes } & \multicolumn{2}{c}{ Método } & \multicolumn{2}{c}{ Resultados } & \multicolumn{2}{c}{ Conclusão } \\
\cline { 2 - 13 } Estrutura & $\mathrm{N}$ & $\%$ & $\mathrm{~N}$ & $\%$ & $\mathrm{~N}$ & $\%$ & $\mathrm{~N}$ & $\%$ & $\mathrm{~N}$ & $\%$ & $\mathrm{~N}$ & $\%$ \\
Presente & 8 & 40 & 51 & 75 & 58 & 85,3 & 47 & 69,1 & 56 & 82,4 & 49 & 72,1 \\
Ausente & 12 & 66,2 & 17 & 25 & 10 & 14,7 & 21 & 30,9 & 12 & 17,6 & 19 & 27,9 \\
\hline Total & 68 & 100 & 68 & 100 & 68 & 100 & 68 & 100 & 68 & 100 & 68 & 100 \\
\hline
\end{tabular}

Em todos os artigos analisados predominou a ausência da frase inicial que contextualiza o tema (66,2\%), existindo diferença estatisticamente significante $\left(\chi_{o}^{2}=7,12\right.$ e $\left.\chi_{c}^{2}=3,84\right)$. Os objetivos estavam presentes na grande maioria dos trabalhos $\left(75 \%, \chi_{o}^{2}=17,0 \quad \chi_{c}^{2}=3,84\right)$; quanto aos participantes $(85,3 \%)$, houve predomínio da descrição dos participantes $\left(\chi_{0}^{2}=33,88, \chi_{c}^{2}=\right.$ $3,84)$. O mesmo aconteceu com o método $(69,1 \%$; $\left.\chi_{\mathrm{o}}^{2}=9,94, \chi_{\mathrm{c}}^{2}=3,84\right)$, os resultados $(82,4 \% \mathrm{com}$ $\left.\chi_{0}^{2}=28,47, \chi_{c}^{2}=3,84\right)$ e as conclusões $(72,1 \%$; $\left.\chi_{\mathrm{o}}^{2}=13,23, \quad \chi_{\mathrm{c}}^{2}=3,84\right)$, existindo diferença estatisticamente significante para cada item, o que revela a adequação da maior parte dos resumos e devida avaliação pelos pareceristas.

Na pesquisa sobre Psicologia Forense/Jurídica no banco de dissertações e teses da Capes, os autores encontraram resultados semelhantes no que concerne à ausência de frase inicial $(65,71 \%)$, objetivos (94,1\%), participantes $(64,7 \%)$, material e resultados $(67,64 \%)$ e conclusões $(73,52 \%)$ explicitados. ${ }^{1}$ Existe, entretanto, a necessidade de um maior cuidado dos autores, dos orientadores e pareceristas, com a redação, a elaboração e as informações constantes nos resumos, para que a adequação da estrutura atinja porcentagens maiores, por volta de 95\%. É preciso haver maior rigor, já que esta parte do discurso vai alimentar as bases de dados bibliográficos e deve respeitar, assim, parâmetros como completude e adequação. ${ }^{24}$

A tipologia de trabalho analisado foi definida em quatro categorias: descritiva (57,36\%), experimental (29,41\%), reflexão teórica e não identificada $(13,23 \%)$. A comparação das categorias foi realizada por meio do teste qui- 
quadrado, sendo $\mathrm{p} \leq 0,05$ e n.g.l $=2$. $\mathrm{O}$ resultado encontrado foi estatisticamente significante com $\chi_{0}^{2}=24,88$ e o $\chi_{c}^{2}=5,99$, demonstrando que há predomínio das pesquisas descritivas. Exemplo desta tipologia é o trabalho realizado por Thomas \& Tarr, que apresentaram as percepções de bailarinos frente à dor e sua relação com a lesão no âmbito de sua carreira. ${ }^{17}$

A categoria experimental obteve 29,41\% de artigos na base de dados, como o trabalho realizado por Hui, Chui \& Woo, que num estudo com 111 idosos verificaram os efeitos da dança no estado de saúde durante o período de 12 semanas, sendo avaliados o equilíbrio, a marcha e a qualidade de vida dos participantes, obtendo resultados positivos e significantes. ${ }^{25}$ Cabe salientar que o esperado para o avanço científico é uma maior produção de pesquisas inferenciais (quase e experimentais).

Do total de 68 artigos analisados, sete (10,29\%) foram registrados em trabalhos de reflexão teórica e apenas dois na categoria não identificada (2,94\%), que foi utilizada quando não foi possível saber o tipo de análise utilizada pelo autor na pesquisa pelo conteúdo do resumo, o que certamente é uma limitação de redação do mesmo.

Witter \& Ferreira, em sua pesquisa de produção científica sobre Idoso e leitura na PsycINFO, obtiveram como resultado 23,81\% de trabalhos na categoria pesquisa descritiva e $74,29 \%$ na categoria inferencial (quase experimental e experimental), permitindo concluir que o investimento maior está em pesquisas que viabilizem estabelecer relações diversas entre as variáveis. ${ }^{26} \mathrm{Na}$ pesquisa sobre Psicologia do trânsito, análise de produção de dissertações e teses realizada por Vieira et al., a tipologia de trabalho teórico obteve $60 \%$ das teses de doutorado e $35,7 \%$ de dissertações de mestrado. Já na categoria pesquisa, $64,2 \%$ foram encontrados no mestrado e $40 \%$ no doutorado. Esses resultados demonstraram a existência de correlação negativa entre os trabalhos de mestrado e doutorado, sendo que as pesquisas são o produto esperado no doutorado. ${ }^{27}$
Os resultados quanto à estratégia de pesquisa foram definidos em quatro categorias: de campo $(82,35 \%)$, não identificadas $(7,35 \%)$, documental $(5,88 \%)$ e laboratorial (4,41\%). A maioria de trabalhos realizados em campo revela que, para melhor analisar e comprovar os efeitos da prática da dança, faz-se necessário estar em contato direto com o público-alvo da pesquisa. Exemplo de trabalho de campo foi o produzido por Oliveira, Pivoto \& Vianna ${ }^{28}$ que avaliou o impacto da dança sênior na qualidade de vida de idosos praticantes, por meio da aplicação do SF36 em um grupo de 103 idosos com encontros semanais em um período de quatro meses. Outro estudo foi o de Guimarães et al., que comparou o sono de 101 idosas entre fisicamente ativas e sedentárias em um período de trinta dias. ${ }^{29}$

A categoria não citada foi utilizada quando não foi possível saber o tipo de estratégia utilizada pelo autor na pesquisa pelo conteúdo do resumo, o que certamente é uma limitação de redação do mesmo.

A pesquisa documental é aquela que utiliza documentos sobre as informações já publicadas em relação ao tema. Um exemplo é o trabalho de Borer, que realizou um levantamento de dados sobre a temática atividade física e prevenção de osteoporose em mulheres com a interação de fatores físicos, hormonais e dietéticos. ${ }^{30} \mathrm{Em}$ relação à categoria laboratorial $(4,41 \%)$, descreve um procedimento de investigação mais difícil, porém mais exato no tocante ao conhecimento produzido, em razão das condições de controle e manipulação. Um exemplo é o trabalho de Raymond et al., que ampliou os estudos desenvolvidos sobre o neurofeedback alpha theta por meio da comparação com outro biofeedback, avaliando as melhorias no desempenho de praticantes de dança. ${ }^{31}$

Devido à alta porcentagem de trabalhos de campo $(82,35 \%)$, não foi necessário aplicar o teste estatístico qui-quadrado, sendo observada a predominância deste. Tais resultados são inversamente comparáveis aos resultados da pesquisa de Gouveia et al. sobre a produção 
científica nacional de depressão pós-parto, cujos resultados foram 56,25\% de trabalhos teóricos e $31,25 \%$ de pesquisa de campo, demonstrando a necessidade de trabalhos exploratórios sobre a temática. ${ }^{32}$

A pesquisa de Cusatis Neto \& Santos demonstra resultados favoráveis sobre a produção científica do curso de Fisioterapia da Faculdade do Clube Náutico Mogiano para a categoria "pesquisa de campo", obtendo 55,4\% dos trabalhos analisados entre os anos de 2000 a 2002 e $44,5 \%$ de trabalhos teóricos, demonstrando um crescimento no investimento e interesse de pesquisadores neste tipo de pesquisa no decorrer dos anos, seguindo a tendência científica atual. ${ }^{33}$

A tipologia de análise dos trabalhos foi dividida em quatro categorias: quantitativa $(45,6 \%)$, mista $(27,9 \%)$, qualitativa $(19,1 \%)$ e não identificada $(7,35 \%)$. O teste qui-quadrado foi estatisticamente significante $\left(\chi_{0}^{2}=21,17\right.$ e o $\chi_{c}^{2}=$ 7,81, $\mathrm{p} \leq 0,05$, n.g.l.= 3), com o predomínio da análise quantitativa, como é o exemplo do trabalho de Alpert et al., que realizaram estudo transversal com 13 mulheres idosas com idade média de 68 anos, no qual foi avaliado o impacto da dança jazz no equilíbrio, cognição e humor durante um período de 15 semanas, cujos resultados mostraram melhora significativa no equilíbrio. ${ }^{34}$

$\mathrm{Na}$ categoria mista, exemplo é o trabalho de Song et al., comparando as mudanças nos comportamentos de saúde, motivação e estado funcional de idosos pertencentes e desistentes de um programa de aprimoramento físico por seis meses, utilizando para a coleta de dados entrevistas semiestruturadas, observação participante e testes. ${ }^{35} \mathrm{~A}$ pesquisa qualitativa de D'Alencar et al. realizou análise qualitativa e descritiva sobre o significado da biodança para idosos pertencentes a um grupo de biodança, analisando-se a vivência da biodança na vida desses idosos, suas descobertas e ressignificados. ${ }^{36}$

Existem atualmente duas tendências importantes de pesquisa, cujos paradigmas têm sido usados para caracterizar o estado da investigação: um que se caracteriza pela adoção de uma estratégia de pesquisa modelada nas ciências naturais e baseada em observações empíricas para explicar fatos e fazer previsões (pesquisa experimental), e outro, que advoga uma lógica própria para o estudo dos fenômenos humanos e sociais, procurando as significações dos fatos no contexto concreto em que ocorrem (pesquisa qualitativa). ${ }^{14,37}$ Ambas são válidas e importantes para o desenvolvimento da ciência, tendo em vista suas características metodológicas, vantagens e desvantagens, possibilidades e limites na interpretação e generalização dos dados.

Witter \& Maria, em sua pesquisa Velbice no banco de teses da Capes, encontraram 48,75\% das teses com análise qualitativa, seguidas de 17,5\% com análise mista e 3,75\% com análise quantitativa. ${ }^{37}$ Resultados similares foram encontrados na pesquisa de produção científica sobre Psicologia Forense, sendo 59,38\% qualitativos, seguidos de $37,5 \%$ mistos e $3,12 \%$ de quantitativos, resultado que possivelmente decorre do fato de a área ainda ser emergente. ${ }^{1,38}$

É importante ressaltar que o estudo apresenta limitações, pois avaliou apenas 68 artigos de três bases de dados disponíveis na BVS, sendo realizada uma pesquisa de metaciência na medida que foram estipulados objetivos sobre a comunicação científica e sobre a metodologia empregada na produção científica. Há outros aspectos que poderiam ser analisados, como o conteúdo dos artigos, o embasamento teórico, áreas de conhecimento envolvidas, variáveis analisadas sobre a dança e os idosos, os aspectos biológicos, sociais e psicológicos envolvidos nos estudos e as conclusões obtidas, entre outras variáveis.

O estudo, entretanto, é relevante na medida em que chama a atenção de pesquisadores da área de envelhecimento sobre os avanços e lacunas na temática da dança, idosos e envelhecimento, bem como destaca aspectos importantes da comunicação e produção científicas. Há possibilidade de desenvolver outras pesquisas sobre comunicação e produção científica na área do envelhecimento. 


\section{CONCLUSÃO}

Tendo em vista os dados resultantes da análise da produção científica referente às publicações veiculadas na base de dados da Biblioteca Virtual de Saúde sobre a temática dança e idoso, concluise que os artigos foram a via de publicação mais utilizada. O número de vocábulos do título foi superior aos 12 vocábulos recomendados pelos manuais científicos. Houve predomínio da múltipla autoria, confirmando a tendência mundial, o que indica bom índice de desenvolvimento nas áreas, sem especificação de gênero, demonstrando que tanto homens como mulheres estão produzindo sobre idosos e dança. O tipo de pesquisa mais observado foi a descritiva, demonstrando

\section{REFERÊNCIAS}

1. Witter GP, Buriti, MA. Psicologia Forense/Jurídica no banco de dissertações e teses da Capes. In: Witter C, Buriti MA, Witter GP, organizadores. Problemas psicossociais: análise de produção. São Paulo: Anadarco; 2007. p. 13-33.

2. Instituto Brasileiro de Geografia e Estatística. Perfil dos idosos responsáveis pelos domicílios no Brasil. [acesso 2011]. Disponível em: http://www.ibge.com.br/ home/estatistica/populacao/perfilidoso/default.shtm.

3. Veras R. Envelhecimento populacional contemporâneo: demandas, desafios e inovações. Rev Saúde Pública 2003;43(3):548-54.

4. Cipriani NCS, Meurer ST, Benedetti TRB, Lopes MA. Aptidão funcional de idosas praticantes de atividades físicas. Rev Bras Cineantropom Desempenho Hum 2010;12(2):106-11.

5. Pena N, Barbosa JL, Fraga JCL, Mattar M, Leite C. Eficácia de um programa de dança para prevenir quedas entre idosos. Rev Baiana Saúde Pública 2008;32(2):168-76.

6. Santos FH, Andrade VM, Bueno OFA. Envelhecimento: um processo multifatorial. Psicol Estud 2009;14(1):3-10.

7. Bocalini DS, Santos RN, Miranda MLJ. Efeitos da prática de dança de salão na aptidão funcional de mulheres idosas. Ciênc Movim 2007;15(3):23-9.

8. de Jong J, Lemmink KA, King AC, Huisman M, Stevens M. Twelve-month effects of the Groningen active living model (GALM) on physical activity, health and fitness outcomes in sedentary and underactive older adults aged 55-65. Patient Educ Couns 2007;66(2):167-76. defasagem de conhecimentos científicos sobre o tema proposto, com a realização de estudos com delineamentos inferenciais. $\mathrm{O}$ tipo de análise mais utilizada foi a quantitativa.

Ficou clara a necessidade de se realizar novas pesquisas sobre a dança, sobretudo no âmbito nacional, para que a população idosa possa se beneficiar de programas e/ou atividades que possibilitem a participação social, estimulem o desenvolvimento das aptidões funcionais e, principalmente, possam incentivar as habilidades biopsicossociais, por meio do conhecimento das habilidades e limitações individuais e de atividades que incentivem a autonomia e o exercício da cidadania. ${ }^{28,29,31,36}$

9. Catib NOM, Schwartz GM, Christofoletti DFA Santiago DRP, Caparroz GP. Estados emocionais de idosos nas danças circulares. Motriz Rev Educ Fis 2008;14(1):41-52.

10. Fonseca CC. Análise do esquema corporal e imagem corporal na dança de salão e seus aspectos motivacionais [dissertação de mestrado]. São Paulo: Universidade São Judas Tadeu; 2008.

11. Tavares MCGC. A imagem corporal e a dança. Conexões 2001;1(6):10-22.

12. Micheli LJ. Back injuries in dancers. Clin Sports Med 1983;2(3):473-84.

13. Holmerová I, Machácová K, Vanková H, Veleta P, Jurasková B, Hrnciariková D, et al. Effect of the exercise dance for seniors program on lower-body functioning among institutionalized older adults. J Aging Health 2010;22(1):106-19.

14. Costa AS, Ferreira AA. Depressão em adolescentes na Psycinfo. In: Witter C, Buriti MA, Witter GP, organizadores. Problemas psicossociais: análise de produção. São Paulo: Anadarco; 2007. p. 103-27.

15. Farias AS, Martins DS, Faria FDM, Witter C. O Normal e o patológico na teoria de D.W. Winnicott. In: Witter C, Buriti MA, Witter GP, organizadores. Problemas psicossociais: análise de produção. São Paulo: Anadarco; 2007. p. 147-70.

16. Ferreira AA, Theodório DP. Estresse em Estudantes Universitários: análise de produção científica. In: Witter GP, organizador. Metaciência e Psicologia. Campinas: Alínea; 2005. p.185-98. 
17. Thomas T, Tarr J. Dancer's perceptions of pain and injury: positive and negative effects. J Dance Med Sci 2009;13(2):51-9.

18. McKinley P, Jacobson A, Leroux A, Bednarczyk V, Rossignol M, Fung J. Effect of a community-based Argentine tango dance program on functional balance and confidence in older adults. J Aging Phys Act 2008;16(4):435-53.

19. Belardinelli R, Lacalaprice F, Ventrella C, Volpe L, Faccenda E. Waltz dancing in patients with chronic heart failure: new form of exercise training. Circ Heart Fail 2008;1(2):107-14.

20. Witter GP. Leitura no Reading Research Quarterly (2000/2002). In: Witter GP, organizador. Metaciência e Psicologia. Campinas: Alínea; 2005. p.137-54.

21. Fernandes MAH. Intervenção em instituição de idosos: ouvir músicas, dançar, escutar histórias, brincar, criar personagens. Psikhe 2003;8(1):7-12.

22. Murrock CJ, Madigan E. Self-efficacy and social support as mediators between culturally specific dance and lifestyle physical activity. Res Theory Nurs Pract 2008;22(3):192-204.

23. Rohleder N, Beulen S, Chen E, Wolf JM, Kirschbaum C. Stress on the floor: the cortisol stress response to social evaluative threat in ballroom dancers. Pers Soc Psychol Bull 2007;33(1):69-84.

24. Silva MS, Ramos LMVC, Noronha DP. Base de Dados. In: Poblacion DA, Witter GP, Silva JFM, organizadores. Comunicação e produção científica: contexto, indicadores e avaliação. São Paulo: Angellara; 2006. p. 261-86.

25. Hui E, Chui BT, Woo J. Effects of dance on physical and psychological well-being in older persons. Arch Gerontol Geriatr 2009;49(1):45-50.

26. Witter GP, Ferreira AA. Idoso e leitura: análise de produção científica arrolada na PsycINFO (2001/2003). In: Witter GP, organizador. Metaciência e Psicologia. Campinas: Alínea; 2005. p. 155-69.

27. Vieira AO, Ferreira AA, Buriti MA, Witter C. Psicologia do trânsito: análise da produção de dissertações teses (1999-2003). In: Witter C, Buriti MA, Witter GP, organizadores. Problemas psicossociais: análise de produção. São Paulo: Anadarco; 2007. p. 51-84.
28. Oliveira LC, Pivoto EA, Vianna PCP. Análise dos resultados de qualidade de vida em idosos praticantes de dança sênior através do SF-36. Acta Fisiatr 2009;16(3):101-04.

29. Guimarães LHCT, Carvalho LBC, Yanaquibashi G, Prado GF. Physically active elderly women sleep more and better than sedentary women. Sleep Med 2008;9(5):488-93.

30. Borer KT. Physical activity in the prevention and amelioration of osteoporosis in women: interaction of mechanical, hormonal and dietary factors. Sports Med 2005;35(9):779-830.

31. Raymond J, Sajid I, Parkinson LA, Gruzelier JH. Biofeedback and dance performance: a preliminary investigation. Appl Psychophysiol Biofeedback 2005;30(1):64-73.

32. Gouvêa AM, Modesto EL, Reis TM, Gatti AL. Depressão pós-parto: produção científica nacional entre 1999 e 2005. In: Witter C, Buriti MA, Witter GP, organizadores. Problemas psicossociais: análise de produção. São Paulo: Anadarco; 2007. p. 171-99.

33. Cusatis Neto R, Santos RS. Produção científica do curso de Fisioterapia da Faculdade do Clube Náutico Mogiano. In: Witter GP, organizador. Metaciência e Psicologia. Campinas: Alínea; 2005. p. 217-36.

34. Alpert PT, Miller SK, Wallmann H, Havey R, Cross C, Chevalia T, Gillis CB, Kodandapari K. The effect of modified jazz dance on balance, cognition and mood in older adults. J Am Acad Nurse Pract 2009;21(2):108-15.

35. Song R, June KJ, Kim CG, Jeon MY. Comparisons of motivation, health behaviors and functional status among elders in residential homes in Korea. Public Health Nurs 2004;21(4):361-71.

36. D’Alencar BP, Mendes MMR, Jorge MSB, Guimarães JMX. Biodança como processo de renovação existencial do idoso. Rev Bras Enferm 2008;61(5):608-14.

37. Witter GP, Maria FA. Velhice no banco de teses da Capes (2000 e 2001). In: Witter GP, organizador. Metaciência e Psicologia. Campinas: Alínea; 2005. p. 171-84.

38. Phelippe HR, Witter GP, Buriti MA. Análise de produção científica sobre Psicologia Forense. IN: Witter C, Buriti MA, Witter GP, organizadores. Problemas psicossociais: análise de produção. São Paulo: Anadarco; 2007. p. 35-50. 